\title{
Concurrent Design of Energy Management and Vehicle Traction Supervisory Control Algorithms for Parallel Hybrid Electric Vehicles
}

\author{
Halil I. Dokuyucu and Melih Cakmakci, Member, IEEE
}

\begin{abstract}
In this paper, concurrent design of energy management (EM) and traction control algorithms for a vehicle equipped with a parallel hybrid powertrain is studied. This paper focuses on designing the two control algorithms together as one control design problem, which are traditionally considered separately. First, optimal control actions and operating points are obtained by applying dynamic programming (DP). Then, this information is used for developing a rule-based supervisory controller. Our objective is to minimize the fuel consumption and the wheel slip simultaneously. Two control problems are also solved separately and compared with the concurrent solution. Results show that promising benefits can be obtained by using the concurrent design approach rather than considering two control problems separately. Under the same conditions, the vehicle with the concurrent supervisory controller is $\mathbf{1 6 \%}$ more efficient in fuel consumption and experiences $12 \%$ less wheel slip, assuming slippery road friction conditions.
\end{abstract}

Index Terms-Concurrent controllers, hybrid electric vehicles (HEVs).

\section{INTRODUCTION}

A UTOMOTIVE companies always search for alternative ways of operating vehicles in more efficient ways to cope with the high performance standards in today's globally competitive market environment. Using hybrid powertrains, which combine two or more power sources, significant improvements in fuel efficiency and emissions are possible. However, the inclusion of the new power sources and the accompanying energy storage systems increase the complexity of the system, bringing new design challenges.

Operation of modern vehicles involves many different systems working together. A typical layout for a hybrid electric vehicle (HEV) controller network is shown in Fig. 1. The vehicle control network has many controllers exchanging important information with each other regarding their component's current state or demand levels. The engine speed information from the engine controller, battery state of charge (SOC) information from the battery controller, or the vehicle torque arbitration commands from the supervisory controller are examples of

Manuscript received March 30, 2014; revised August 20, 2014 and October 21, 2014; accepted February 16, 2015. Date of publication February 19, 2015; date of current version February 9, 2016. The review of this paper was coordinated by Prof. T. Shim.

The authors are with the Department of Mechanical Engineering, Bilkent University, 06800 Ankara, Turkey (e-mail: dokuyucu@bilkent.edu.tr; melihc@ bilkent.edu.tr).

Color versions of one or more of the figures in this paper are available online at http://ieeexplore.ieee.org.

Digital Object Identifier 10.1109/TVT.2015.2405347

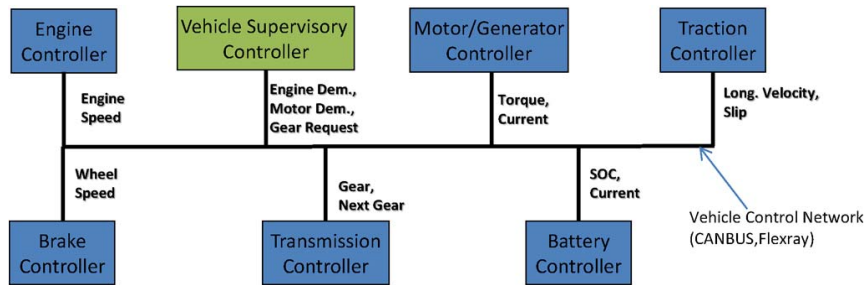

Fig. 1. Typical automotive controller network for an HEV.

these communications. The information from the network is typically used in ad hoc control algorithms added to the baseline component controller algorithms. These algorithms are developed either without the external information or with the constant value assumptions (i.e., using an average SOC value, engine speed, etc.) earlier in the vehicle design cycle.

Design and performance related to energy management (EM) and vehicle dynamics controllers are a very popular topic in automotive control literature. The two topics are usually considered and investigated separately. For both studies on EM and vehicle dynamics, once the optimal control trace is obtained, a casual control algorithm is designed as the second step to complete the strategy development with dynamic programming (DP) [1]-[5].

In [3], optimal EM strategies are studied. In [6], minimum fuel consumption is evaluated considering the optimal control theory. In [7], optimizing the fuel economy and balancing the state of charge of the battery is studied. In [4], [8], and [9], the DP method is used to obtain the optimal strategy for HEVs. Traditionally, EM strategies for HEVs are developed considering the powertrain dynamics only [1], [2], [6], [10], [11]. Recently, in [12], a comparison of three known methods for solving the resulting optimization problem, i.e., DP, Pontryagin's minimum principle (PMP), and the equivalent consumption minimization strategy (ECMS), are described and analyzed. It is reported that, of the three strategies, ECMS is the only implementable in real time; the equivalence with PMP and DP justifies its use as an optimal strategy and allows more effective tuning. The state of charge (SOC) of the battery is used extensively in supervisory control algorithms to influence the vehicle behavior. In [13], a direct mathematical approach is used to determine the SOCdependent equivalent cost factor in HEV supervisory control problems using the globally optimal DP. This study is then used to design cost minimization strategies, which achieve near optimal fuel economy. 
DP techniques are also used in vehicle stability control development process. In [1], DP is applied to the vehicle stability problem by giving all the power of electric motor to the rear axle and all the power of the internal combustion engine to the front axle. The use of case transfer device to improve stability is also considered. In [14], a control strategy that optimizes vehicle performance while guaranteeing vehicle stability and drivability is developed by actively controlling the transfer case. The use of desired slip parameter is common approach for many traction control algorithms. In [15], a second-order slidingmode traction controller is coupled with the design of a suitable sliding-mode observer to estimate the tire-road adhesion coefficient. The traction control is achieved by maintaining the wheel slip at a desired value by controlling the wheel slip at the optimal value, improving safety under difficult weather conditions. In [16], a new torque control method for various drive-slip conditions involving abrupt changes in the road friction. This method is based on a proportional-integral-differential (PID) plus fuzzy logic controller for driving torque regulation, which consists of a PID controller and a fuzzy logic controller. In [17], an adaptive gradient ascent algorithm is used for longitudinal traction control. A real-time implementation of the gradient ascent algorithm is developed using linear operator techniques, although the tire-ground interface is highly nonlinear. In [18], the lower level controller, i.e., a wheel slip controller, is designed to keep the slip ratio at each wheel below a limit value. In addition, an optimization-based control allocation strategy is used to map the upper level and wheel slip control inputs to actual actuator commands, taking into account the actuator constraints.

In this paper, a method developed for concurrent design of control algorithms is presented as the main contribution. This paper is presented using two interacting control problems from the automotive design field. Two control systems are operated together using a combined supervisory controller, and improvements are investigated using simulations. As the interaction between these two problems grows, significant improvements in the terms of fuel consumption and wheel slip behavior can be achieved when the control problems are solved together. The effect of coupling in control problems is also observed in [19]. The strong interaction between these two control problems is outlined in [20]. The remainder of this paper is organized as follows: In Section II, vehicle models used for controller development and controller verification are explained. The DP problem solved for EM and traction control algorithms (concurrently and separately) is given in Section III. The supervisory controller developed based on the optimal operation points obtained in Section III is presented in Section IV with simulation results. Conclusion and future work is given in Section V.

\section{Vehicle Configuration And Models}

Fig. 2 shows the powertrain layout of the parallel HEV (PHEV) used in this paper. For the typical PHEV configuration, engine and electric motor torque are coupled using the so-called power split transmission system. The total torque can be distributed to the front and rear wheels by using the transfer case component in all-wheel-drive systems. The electric motor has the capability of working as a generator to charge the electric

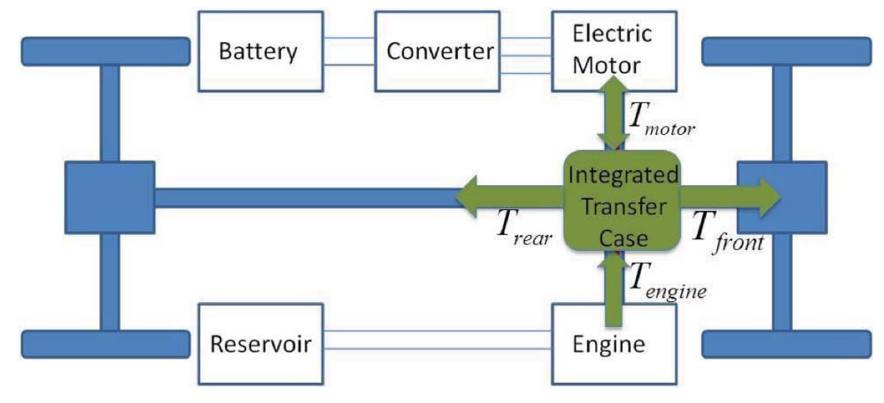

Fig. 2. PHEV powertrain layout.

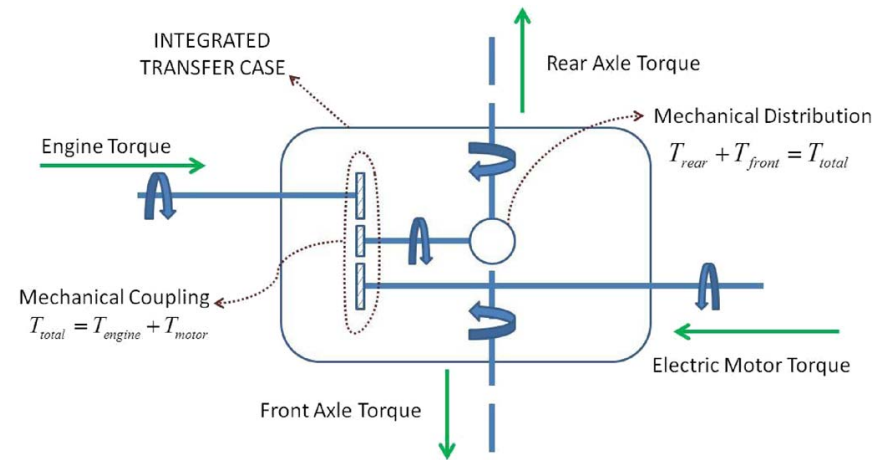

Fig. 3. Integrated transfer case unit.

battery. The parallel hybrid powertrain configuration is more general compared with series hybrid powertrain configuration since it has more operating modes.

For this paper, an integrated (i.e., combined power split and transfer case) transfer case component is used as shown in Fig. 3. The total torque demand of the vehicle is delivered by the electric motor $\left(T_{\text {motor }}\right)$ and the engine $\left(T_{\text {engine }}\right)$ combined by the power-split transmission. A transfer case unit distributes the total torque between the rear $\left(T_{\text {rear }}\right)$ and the front axles $\left(T_{\text {front }}\right)$. The torque split between rear and front axles is used to provide stability during acceleration and braking of the vehicle.

For solving the DP problem, a simplified vehicle model is developed as outlined in Section II-A. The simple model is suitable for studying both the EM and vehicle stability problems together and separately. A PHEV model in Simulink is also developed using the Autonomie software (see Section II-B) library. This is a complex simulation model based on empirical vehicle data driven by realistic control algorithms and a driver algorithm. We used the complex model for verification of the supervisory control strategies. Same vehicle parameters are used for both the simple and complex models. These parameters are given in Table I.

\section{A. Simple Vehicle Model}

A simple but functional mathematical model is developed suitable for a DP formulation. By solving this DP problem, the optimal operating points of the powertrain configuration are investigated. The simplified model has two dynamic states: the SOC of the battery SOC and the wheel slip $\lambda$. The formulation is detailed enough that the same model can be used in all of the DP studies. This is important for the fair comparability of the results. 
TABLE I

Vehicle PARAmeters

\begin{tabular}{|l|l|}
\hline Component & Component Parameters \\
\hline Internal Combustion & 4 cylinders \\
Engine (SI) & 2.2 liters \\
& $84 \mathrm{~kW}$ (peak power) \\
& $250 \mathrm{~kg}$ (mass) \\
\hline Permanent Magnet & $53 \mathrm{~kW}$ (peak power) \\
Electric Motor (brushless) & $248 \mathrm{~N} . \mathrm{m}$ (peak torque) \\
\hline Nimh Ovonic Battery & 28 Ah (capacity) \\
& 50 (number of modules) \\
& 6 V/module (nominal voltage) \\
& $48.6 \mathrm{Wh} / \mathrm{kg}$ (Energy density) \\
& $444.4 \mathrm{~W} / \mathrm{kg}$ (Power density) \\
\hline Vehicle Body & Mass: $800 \mathrm{~kg}$ \\
& Wheel radius: $0.27 \mathrm{~m}$ \\
& Veh. Drag Coeff.: $0.48 \mathrm{Ns}^{2} / \mathrm{m}^{2}$ \\
& Surf. Fric. Coeff.: 0.3 (wet road) \\
\hline
\end{tabular}

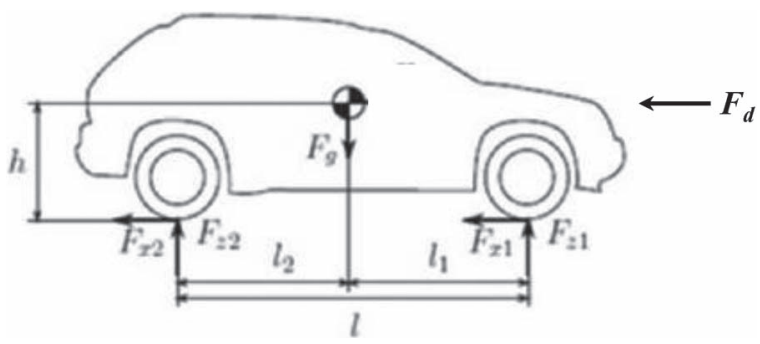

Fig. 4. Free-body diagram for the vehicle model.

The vehicle motion is modeled including both the rear and front wheel axles without considering the lateral dynamics. Similar models can be found in many automotive-controlrelated references such as in [11]. The weight transfer between front and rear axles is considered due to the vehicle acceleration. The Free-body diagram of the vehicle is shown in Fig. 4. $F_{z 1}$ and $F_{z 2}$ denote the vehicle traction forces of the front and rear wheels, respectively. $F_{g}$ represents the weight of the vehicle, and $F_{L}$ represents the inertial force acting the vehicle. $l$ is used for the distance between the axles of the vehicle. $l_{1}$ is the horizontal distance between the center of gravity of the vehicle and the front axle, and $l_{2}$ is the horizontal distance between the center of mass of the vehicle and the rear axle. $h$ is the vertical distance of the center of mass from the ground.

In the case of static equilibrium, the reaction forces $F_{z 1}$ and $F_{z 2}$ at the contact points between tires and the road can be given as

$$
F_{g}=m g=F_{z 1}+F_{z 2}
$$

where

$$
F_{z 1}=m g \frac{l_{2}}{l}, \quad F_{z 2}=m g \frac{l_{1}}{l} .
$$

$F_{x 1}$ and $F_{x 1}$ are the longitudinal tire forces acting on the vehicle. These forces are limited due the friction between the tire and the road surface. This limit is calculated in the following using the uniform friction coefficient $\mu$ :

$$
\left|F_{x i}\right| \leq\left|F_{x i, \max }\right|=\mu F_{z i} \quad \text { for } i=1,2
$$

where $F_{x i}$ is the actual wheel force, and $F_{x i, \max }$ is the maximum value of this force due to friction.
The relationship between the vehicle acceleration and the longitudinal tire force can be obtained as

$$
a_{\mathrm{veh}}=\frac{F_{x 1}+F_{x 2}-F_{d}}{m}=\frac{F_{x}}{m}
$$

where $F_{d}$ is the vehicle drag force changing with respect to the longitudinal velocity, and $F_{x}$ is the net longitudinal tire force, which is limited by the maximum longitudinal forces.

Weight transfer of vehicle during acceleration (or deceleration) is modeled as shown in

$$
\begin{aligned}
& F_{z 1}=F_{g} \frac{l-l_{1}}{l}-\frac{\left(m a_{\mathrm{veh}}+F_{d}\right) \times h}{l} \\
& F_{z 2}=F_{g} \frac{l_{1}}{l}+\frac{\left(m a_{\mathrm{veh}}+F_{d}\right) \times h}{l} .
\end{aligned}
$$

In (5) and (6), the first terms after the equality represent the static weight distribution, and the second terms represent the dynamic weight distribution due to acceleration or deceleration of the vehicle. Using the transfer case component discussed earlier, front and rear torque values are distributed using the torque split factor (TSF), as shown in the following:

$$
\begin{aligned}
T_{\text {front }} & =T_{\text {total }} \times(1-\mathrm{TSF}) \\
T_{\text {rear }} & =T_{\text {total }} \times \mathrm{TSF} .
\end{aligned}
$$

The rear rotational speed ratio (RRSR) is a dynamic state of the vehicle model, and it depends on the speed difference between the front and the rear axles. RRSR $\omega_{\text {ratio,rear }}$ can be calculated as a ratio of the rear-axle and front-axle rotational speeds $\omega_{\text {rear }}$ and $\omega_{\text {front }}$, respectively, as shown in the following:

$$
\omega_{\text {ratio,rear }}=0.5+\frac{\omega_{\text {rear }}-\omega_{\text {front }}}{0.5 \times\left(\omega_{\text {rear }}+\omega_{\text {front }}\right)} .
$$

Given $\omega_{\text {ratio,rear }}$, the wheel slip $\lambda$ can be calculated as $\lambda=$ $\omega_{\text {ratio,rear }}-0.5$. The objective of the vehicle traction controller is to regulate the RRSR to minimize slip. When the slip is zero, the vehicle speed $v_{\text {veh }}$ is calculated as $v_{\text {veh }}=\omega_{\text {rear }} r_{w}$, where $r_{w}$ is the effective tire radius.

For the engine, a quasi-static model is used. The 2-D table derived from the actual vehicle data determines fuel consumption rate $m_{f}$ and the theoretical engine torque $T_{\text {eng. The output }}$ torque of the engine, including the frictional power losses of the engine $P_{\text {loss,eng }}$, is calculated as follows:

$$
T_{\text {engine }}=T_{\text {eng }}-\frac{P_{\text {loss }, \text { eng }}}{\omega_{\text {eng }}} .
$$

$P_{\text {loss,eng }}$ is taken as a constant average value in the simple model. A constant fuel injection rate is assumed during the idle speed.

The open-circuit voltage of the battery $V_{\mathrm{oc}}$ can be calculated as

$$
V_{\mathrm{oc}}=\frac{q_{\mathrm{batt}}}{C_{\mathrm{batt}}}
$$

where $q_{\text {batt }}$ represents the charge of the battery. The charge capacity of the battery $C_{\text {batt }}$ depends on the internal temperature and current of the battery. The SOC of the battery is a 
TABLE II

PARAMETERS OF THE ElECTRIC MOTOR

\begin{tabular}{|l|l|}
\hline Parameter & Value \\
\hline$i_{\text {max }, \text { mot }}$ & $475 \mathrm{~A}$ \\
\hline$P_{\text {max }, \text { mot }}$ & $53 \mathrm{~kW}$ \\
\hline$\omega_{\text {max }, \text { mot }}$ & $8000 \mathrm{rpm}$ \\
\hline
\end{tabular}

normalized value, which represents the charge capacity of the battery, i.e.,

$$
\mathrm{SOC}=\frac{V_{\mathrm{oc}}-V_{\min }}{V_{\max }-V_{\min }}
$$

where $V_{\min }$ and $V_{\max }$ represent the minimum and maximum allowable voltages of the battery. These minimum and maximum values are used as 250 and $400 \mathrm{~V}$, respectively, compatible with the electric motor used. The output voltage of the battery can be calculated as

$$
V_{\mathrm{out}}=V_{\mathrm{oc}}-\frac{d q_{\mathrm{batt}}}{d t} R_{\mathrm{batt}}
$$

where $i_{\text {batt }}=d q_{\text {batt }} / d t$ and $R_{\text {batt }}$ are the battery current and the effective battery resistance, respectively. In the simple model, the transients and the thermal effects are neglected.

Electric motor dynamics are faster than battery dynamics. The motor model used for the simple model does not have a dynamic state. The losses of the motor are taken into account considering the output torque and speed. The power exchange by the motor and the battery $P_{\text {batt }}$ is a mapped function of output torque $T_{\text {mot }}$ and speed $\omega_{\text {mot }}$ as shown in the following:

$$
P_{\text {batt }}=P_{\text {map }, \text { motor }}\left(T_{\text {mot }}, \omega_{\text {mot }}\right) .
$$

The maximum allowable current $i_{\max \text { mot }}$ limits the maximum electric power $P_{\max \text {,mot }}$ supplied from the motor as calculated in the following:

$$
P_{\max , \operatorname{mot}}=i_{\max , \operatorname{mot}} V_{\text {out }} .
$$

The mechanical torque limit $T_{\max \text {,mech }}$ is calculated using the peak torque value $T_{\text {peak }}\left(\omega_{\text {mot }}\right)$, the continuous torque $T_{\text {cont }}\left(\omega_{\text {mot }}\right)$, and the motor rotational speed $\omega_{\text {mot }}$, as given in the following:

$T_{\text {max } \text { mech }}=T_{\text {peak }}\left(\omega_{\text {mot }}\right)-\mathrm{HI}\left[T_{\text {peak }}\left(\omega_{\text {mot }}\right)-T_{\text {cont }}\left(\omega_{\text {mot }}\right)\right]$.

The heat index HI is a scaling factor. The maximum allowable output torque of the motor $T_{\max , \text { elec }}$ is given as

$$
T_{\text {max }, \text { elec }}=P_{\text {map }, \text { motor }}^{-1}\left(P_{\text {max }, \text { motor }}, \omega_{\text {mot }}\right)
$$

where $P_{\text {map,mot }}^{-1}$ represents the inverse torque map for the motor. In Table II, maximum limits of the electric motor variables are given.

\section{B. Complex Vehicle Model}

A detailed nonlinear simulation model in Matlab/Simulink environment is developed for testing the developed supervisory controller. This model uses the Autonomie simulation software libraries [21]. The model is configured by selecting the appropriate model library blocks, which are developed based on

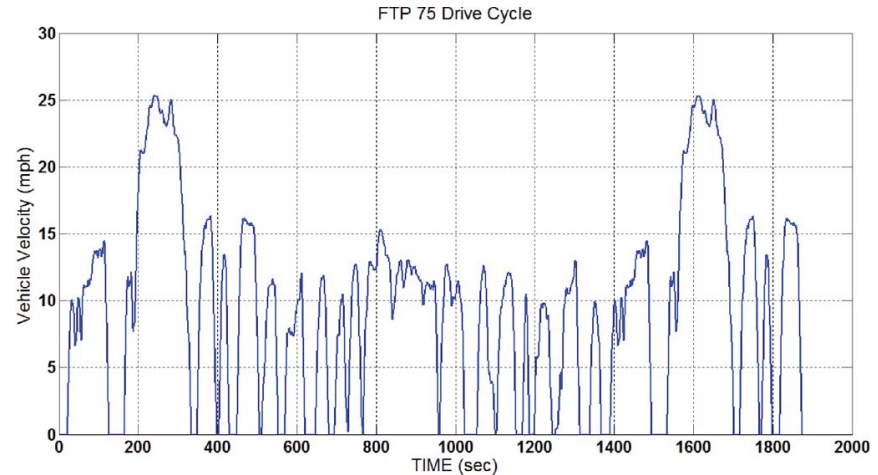

Fig. 5. Urban dynamometer driving schedule (FTP75).

actual data accompanied by realistic control algorithms. The original supervisory controller blocks are modified based on our controller design results. Two power paths provided by the engine and the electric motor are separated. The total torque from the engine and the electric motor is calculated. The total torque is then distributed to the rear and front axles using an integrated transfer case unit model block. The model blocks of the rear and front axles are separated from each other. The sensor information is sent to the controller so that appropriate demand calculations can be made. The simulation model contains a driver block, which selects the appropriate driver commands (acceleration and braking) given a time-based drive cycle.

\section{DYNAMIC PROGRAMMING}

There are various methods to solve a constrained optimization problem. For this paper, DP is used, and the dynamics of the system components are included. Furthermore, with this method, optimization is performed based on the entire demand horizon, which provides globally optimum results. The standard formulation given in [22] is followed. Using the simple parallel hybrid vehicle model developed earlier, DP problems are solved using a specific drive cycle. These optimal results are then used to analyze the optimal operation points of the system to design controllers in the following. The DP problem is solved concurrently (i.e., combined EM and traction control problems) and separately as described in the following. The FTP75 velocity drive cycle shown in Fig. 5 is used for all problems.

\section{A. Concurrent Optimization Problem}

A dynamic optimization problem for the case of EM and vehicle traction control is developed. The two dynamics states, i.e., SOC and RRSR, are used together in the concurrent problem formulation. These dynamic states interact in the concurrent problem formulation, forming a coupled problem. The optimization variables are the torque split ratio (TSR) and TSF, representing the decisions by the EM and traction controllers, respectively. The concurrent DP formulation is given in the following:

$$
\begin{aligned}
& \min _{\left\{u_{k}\right\}}=\left\{\operatorname{TSR}_{k}\left(\left\{x_{k}\right\}\right), \operatorname{TSF}_{k}\left(\left\{x_{k}\right\}\right)\right\} \\
& =\sum_{k=1}^{N-1}\left\{J_{\mathrm{em}, k}\left(\left\{x_{k}\right\},\left\{u_{k}\right\}\right)+J_{\mathrm{tc}, k}\left(\left\{x_{k}\right\},\left\{u_{k}\right\}\right), k\right\}
\end{aligned}
$$


where

$$
\begin{aligned}
x_{k+1}^{1} & =f_{\mathrm{em}}\left(\left\{x_{k}\right\},\left\{u_{k}\right\}\right)+x_{k}^{1}, \quad k=0,1, \ldots, N-1 \\
x_{k+1}^{2} & =f_{\mathrm{tc}}\left(\left\{x_{k}\right\},\left\{u_{k}\right\}\right)+x_{k}^{2}, \quad k=0,1, \ldots, N-1 \\
\left\{x_{0}\right\} & =\{0.5,0.5\} \\
\left\{x_{N}\right\} & =\{0.5,0.5\}
\end{aligned}
$$

subject to

$$
\begin{aligned}
& 0.4 \leq x_{k}^{1} \leq 0.7, \quad-1 \leq u_{k}^{1} \leq 1 \\
& 0.3 \leq x_{k}^{2} \leq 0.7, \quad 0 \leq u_{k}^{2} \leq 1
\end{aligned}
$$

In (18) and (19), shown below, the state vector $\left\{x_{k}\right\}$ is the union of the EM and the traction states, i.e., $\left\{x_{k}\right\}=$ $\left\{\mathrm{SOC}_{k}, \mathrm{RRSR}_{k}\right\}$. Similarly, the controller variables are given as $\left\{u_{k}\right\}=\left\{\mathrm{TSR}_{k}, \mathrm{TSF}_{k}\right\}$.

Based on the formulation presented for the simple model in Section II-A, the calculation of the state equations in (18) can be given as follows: The specific acceleration profile to be followed by the vehicle can be obtained from the predetermined drive cycle information. This acceleration requirement is used to calculate the overall traction force required at the wheels. The force and the acceleration information is then used to calculate the load transfer $F_{z 1}$ and $F_{z 2}$ on the front and rear wheels to check for the vehicle slip conditions. $f_{\mathrm{tc}}$ is the state equation in (9), where the RRSR is calculated using (1)-(9). The wheel traction force calculated can be converted to the traction torque that must be delivered to the wheels from the powertrain system. By using the transmission ratio, the amount of total torque that must be delivered to the power-split case by the engine and the electric motor is calculated. The powersplit ratio (PSR) is used to calculate how much of this torque must come from the engine and the electric motor. By using the operating conditions (torque and rotational speed), the amount of fuel and electricity that must be used by the engine and the motor is calculated, respectively. $f_{\mathrm{em}}$ is the state equation where the current SOC of the vehicle is calculated from using the formulation in (10)-(17). More detailed description of the optimization variables and functions for each case is given in Section III-B and C.

The concurrent problem formulation reduces to the EM problem formulation when the state and control variables of the traction controller problem is kept fixed and vice versa. It is observed that the interaction between the state variables and increased number of controller variables provide better results during the concurrent optimization.

\section{B. Energy Management Problem}

The objective of the EM controller design problem is to find the controller actions that minimizes the fuel consumption of the vehicle over a predefined drive cycle. The simple vehicle model given in Section II-A is used. The SOC is the only dynamic state in the model, and the TSR between the internal combustion engine and the electric motor is the controller variable.
TABLE III

Working Modes of POWER-SPLit Powertrain

\begin{tabular}{|l|l|}
\hline TSR RANGE & MODE \\
\hline$T S R=0$ & Electric Motor Only Mode \\
\hline $0<T S R<1$ & Torque Assist Mode \\
\hline$T S R=1$ & Engine Only Mode \\
\hline $1<T S R$ & Battery Charging Mode \\
\hline
\end{tabular}

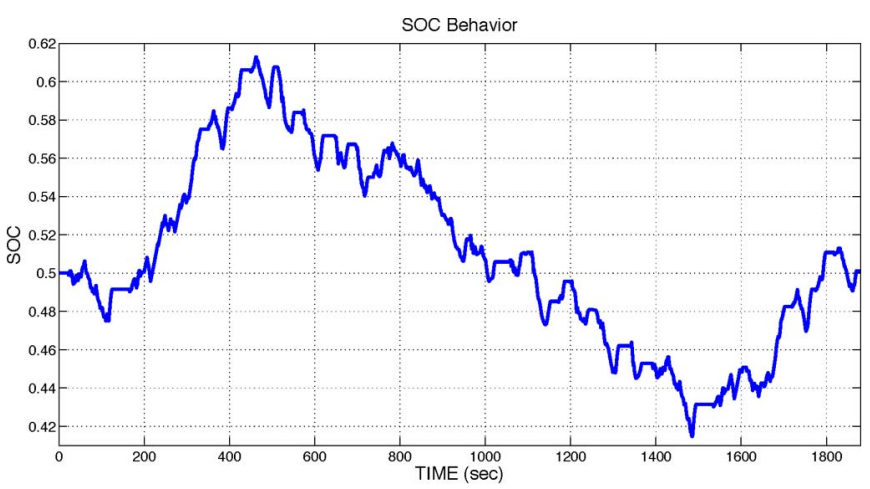

Fig. 6. SOC of the battery with the EM controller.

The optimization problem for EM controller is formulated as shown in the following:

$$
\min _{\mathrm{TSR}_{k}} J=\sum_{k=1}^{N-1} J_{\mathrm{em}, k}\left(\mathrm{SOC}_{k}, \mathrm{TSR}_{k}\right)
$$

where

$$
\mathrm{SOC}_{k+1}=f_{\mathrm{em}}\left(\mathrm{SOC}_{k}, \mathrm{TSR}_{k}\right)
$$

subject to

$$
\begin{aligned}
\mathrm{SOC}_{0} & =0.5 \\
\operatorname{SOC}_{N} & =0.5 \\
0.4 & \leq \mathrm{SOC}_{k} \leq 0.7, \quad-1 \leq \mathrm{TSR}_{k} \leq 1 .
\end{aligned}
$$

In (20), $f_{\mathrm{em}}\left(\mathrm{SOC}_{k}, \mathrm{TSR}_{k}\right)$ is a function used for calculating the current state based on the simple model formulation. Since we are only concerned with the EM side of the problem, the RRSR is taken as constant $\left(\mathrm{RRSR}=x_{k}^{2}=0.5\right)$. Similarly, a constant TSF $\left(\mathrm{TSF}=u_{k}^{2}=0.5\right)$ is also used. $J_{\mathrm{em}}\left(\mathrm{SOC}_{k}, \mathrm{TSR}_{k}\right)$ is the fuel consumption of the PHEV as calculated in the following:

$$
J_{\mathrm{em}}\left(\mathrm{SOC}_{k}, \mathrm{TSR}_{k}\right)=\Delta m_{f}\left(\mathrm{SOC}_{k}, \mathrm{TSR}_{k}, k\right) \times T_{s} .
$$

$T_{s}$ is the simulation step size. The engine fuel rate $\Delta m_{f}$ is calculated by using a predefined table from current operation conditions. $N$ denotes the number of calculation steps in the DP procedure based on the length of the drive cycle. TSR is defined as

$$
\mathrm{TSR}=\frac{\text { Engine Torque Calculated at the Wheels }}{\text { Total Torque Calculated at the Wheels }} .
$$

The operation modes of the hybrid powertrain based on the TSR value are given in Table III.

The optimal TSR over the drive cycle is found by solving the DP problem given in (19)-(23). In Fig. 6, the optimal SOC 


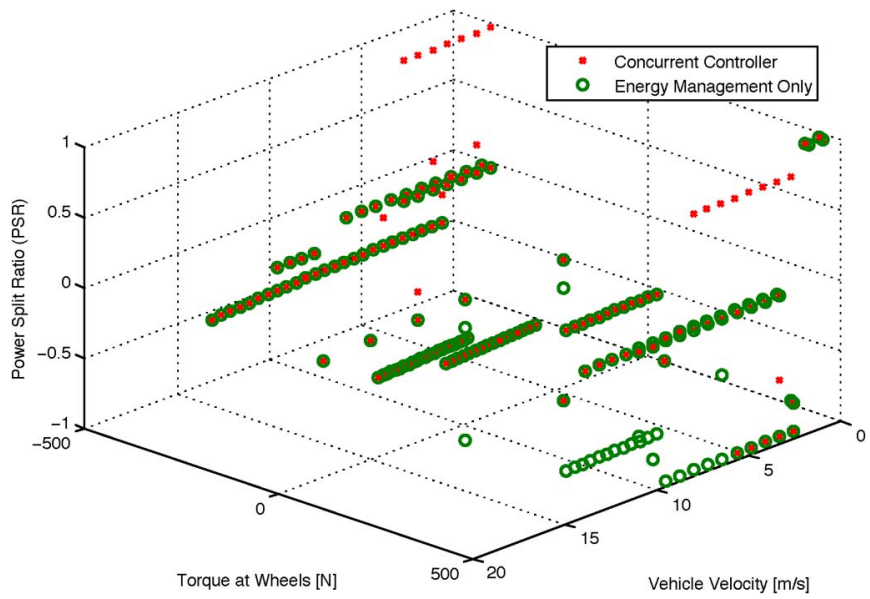

Fig. 7. Optimal operating points of EM versus concurrent controllers.

solution is presented. In Fig. 7, the optimal PSR values are plotted against the torque demand to find the optimal operating points of the system. The power-split operating modes of the vehicle based on desired torque at wheels and vehicle velocity is given in Fig. 7. The results in Fig. 7 show that the vehicle is working in the electric-motor-only mode in the low torque demand range when the vehicle is launched (low velocity and torque demand). Except during vehicle launch, recharging mode is preferred for the low torque demand range. Engine only mode is dominant in the middle torque demand range, and the torque assist mode is preferred in the high-torquedemand region. The concurrent problem optimal data points in Fig. 7 show a similar trend with a broader torque demand span except in low speed charging and high torque demand regions, where in the concurrent case, the effect of vehicle slip is also considered.

\section{Traction Control Problem}

The objective of the vehicle traction controller problem is to minimize the wheel slip of the vehicle over a predefined drive cycle. The RRSR $\omega_{\text {ratio,rear }}$ is the only dynamic state. The TSF between front and rear axles is used as the control variable. The friction coefficient is assumed constant for the full drive cycle. The traction control DP problem is given in the following:

$$
\min _{\mathrm{TSF}_{k}} J=\sum_{k=1}^{N-1} J_{\mathrm{tc}, k}\left(\mathrm{RRSR}_{k}, \mathrm{TSF}_{k}\right)
$$

where

$$
\operatorname{RRSR}_{k+1}=f_{\mathrm{tc}}\left(\mathrm{RRSR}_{k}, \mathrm{TSF}_{k}\right)
$$

subject to

$$
\begin{aligned}
\operatorname{RRSR}_{0} & =0.5 \\
\operatorname{RRSR}_{N} & =0.5 \\
0.3 & \leq \operatorname{RRSR}_{k} \leq 0.7, \quad 0 \leq \mathrm{TSF}_{k} \leq 1 .
\end{aligned}
$$

In (26), $f_{\mathrm{tc}}\left(\mathrm{RRSR}_{\mathrm{TSF}}\right)$ is a function used for calculating the current state based on the simple model formulation. Initial and final values for the TSF are both taken as 0.5. Since we are
TABLE IV

Working Modes of THE TRAnsfer CASE

\begin{tabular}{|l|l|}
\hline TSF RANGE & MODE \\
\hline$T S F=0$ & Rear Axle Only Mode \\
\hline $0<T S F<1$ & Front and Rear Mixing Mode \\
\hline$T S F=1$ & Front Axle Only Mode \\
\hline
\end{tabular}

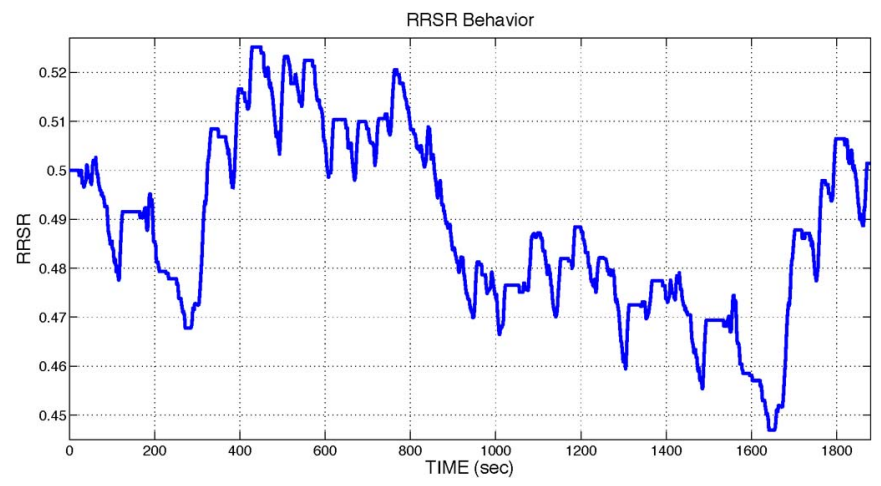

Fig. 8. RRSR behavior of the traction controller.

only concerned with the traction control side of the problem, no electric motor activity is used. The SOC is taken as a nominal constant $\left(\mathrm{SOC}=x_{k}^{1}=0.5\right)$. Similarly, all engine TSR $\left(\mathrm{TSR}=u_{k}^{2}=1\right)$ is used. $J_{\mathrm{tc}}\left(\mathrm{RRSR}, \mathrm{TSF}_{k}\right)$ is the wheel slip of the PHEV as shown in

$$
J_{\mathrm{tc}}\left(\mathrm{RRSR}_{k}, \mathrm{TSF}_{k}\right)=\Delta \lambda\left(\mathrm{RRSR}_{k}, \mathrm{TSF}_{k}, k\right) \times T_{s} .
$$

TSF is defined as

$$
\mathrm{TSF}=\frac{\text { Front Axle Torque Calculated at Wheels }}{\text { Total Torque Calculated at Wheels }} .
$$

The operating modes of the transfer case based on the TSF are given in Table IV.

The optimal RRSR trace is given in Fig. 8, showing a highly active use of the transfer case in the mixed torque transfer mode. In Fig. 9, the optimal TSF points are plotted against vehicle speed and the torque demand in order to present the optimal operating points. At the low speed range, rear-axle-only mode is preferred. Front-and-rear-axle mixing mode is dominant in the middle and high speed ranges in the presence of torque demand. Fig. 9 also shows the optimal traces of the concurrent controller. Torque assist mode is more dominant in the high torque demand range. It is important to note that the difference in operating points occur particularly for the high-torque-demand areas that are prone to most slipping instances for the vehicle.

In Figs. 10 and 11, the fuel rate and the wheel slip comparison are given for the individual and concurrent cases. The fuel rate is decreased when the concurrent controller is used since energy loss due to the slip is also minimized. The concurrent controller operates the engine at the optimum fuel rate by making use of the torque assist mode.

The total fuel and the wheel slip calculated over the FTP75 drive cycle given in Table V. The results indicate that high levels of fuel efficiency can be obtained for the long-range driving conditions. Sample hard acceleration and braking ranges are highlighted. Less wheel slip is observed for the same 


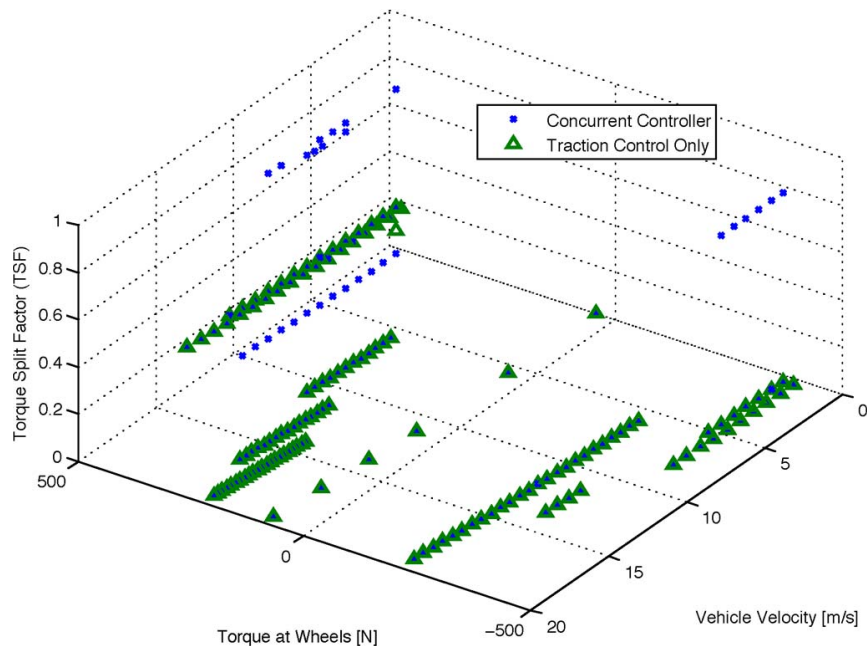

Fig. 9. Optimal operating points of the traction and the concurrent controllers.

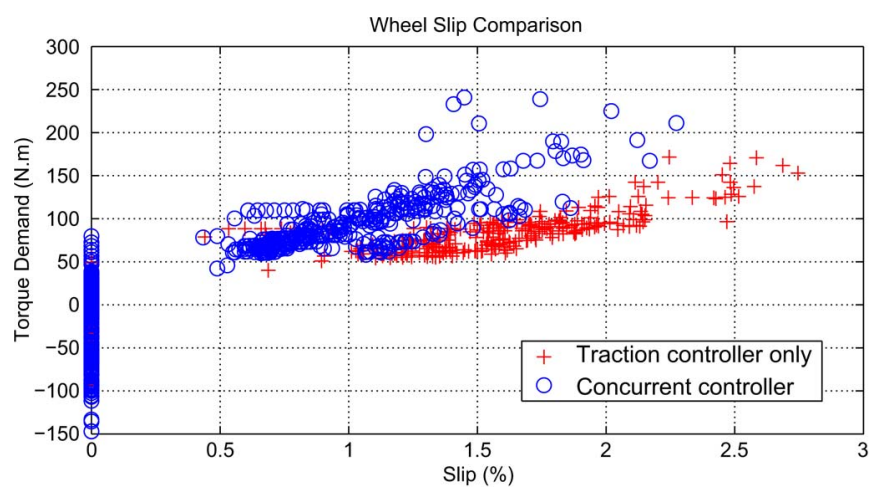

Fig. 10. Wheel slip comparison of traction stability and concurrent controllers.
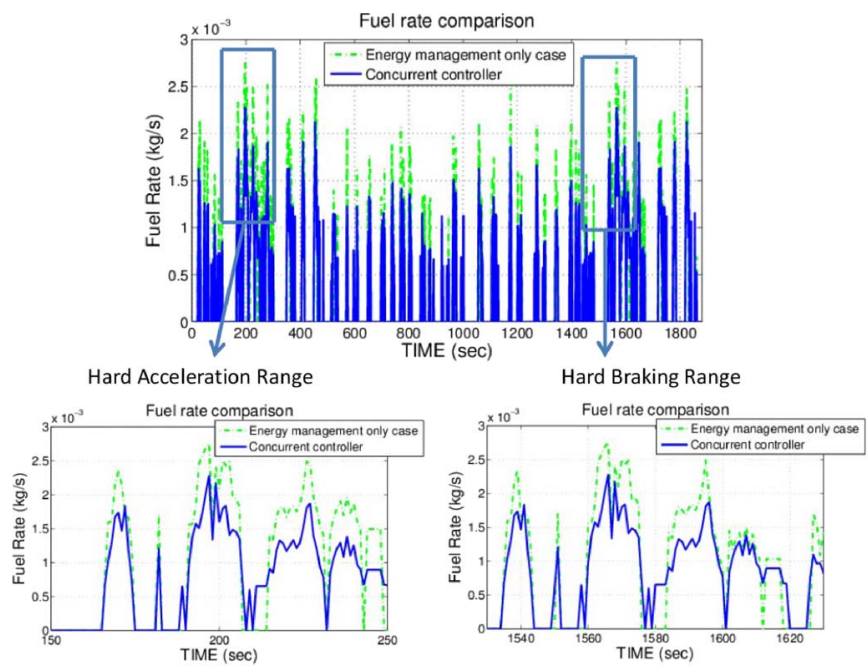

Fig. 11. Fuel rate comparison of concurrent and EM controllers.

torque demand when concurrent controller is used. This is because the traction controller and the EM algorithms simultaneously in effect during hard acceleration and braking regions.
TABLE $\mathrm{V}$

FUEL CONSUMPTION AND WHEEL SLIP COMPARISON OVER FTP75 CYCLE

\begin{tabular}{|l|c|c|c|c|}
\hline & Fuel & Average & \multicolumn{2}{|c|}{ Improvement } \\
& $\begin{array}{c}\text { Cons. } \\
(l / 100 \mathrm{~km})\end{array}$ & $\begin{array}{c}\text { Wheel Slip } \\
(\%)\end{array}$ & $\begin{array}{c}\text { Fuel } \\
\text { Cons. }\end{array}$ & $\begin{array}{c}\text { Average } \\
\text { Wheel Slip }\end{array}$ \\
\hline EM & 8.3 & & & \\
\hline TC & & 1.7 & & \\
\hline Concurrent & 7.5 & 1.4 & $9.63 \%$ & $17.6 \%$ \\
\hline
\end{tabular}

\section{Supervisory Controller DeVelopment}

After the optimal control actions and the operating points of the system are identified using DP, supervisory controllers that operate the vehicle systems are designed. It should be noted that developing a real-time controller designed by extracting parameters from the optimal plant and controller response after solving the DP problem would be only valid for the drive cycle used. This approach may be useful for some repetitive task applications such as path planning in manufacturing processes; it is not useful for automotive applications where the drive profile is not constant. Instead, our supervisory controller development method follows the steps of identifying the optimal operation states where the vehicle is most efficient and then of designing algorithms that steer the vehicle states near these optimal points during actual operation.

The vehicle model is then simulated against the Urban Dynamometer Driving Schedule (UDDS) with the controllers. Results of these simulations are presented here.

There are two split mechanisms in the powertrain: 1) power split between the electric motor and the engine determined by EM controller using the PSR; 2) torque distribution between the front and the rear axles regulated by traction controller using the TSF. PSR is primarily influenced by the SOC of the vehicle, and TSF is primarily determined by the amount of vehicle slip. Optimal operating points based on vehicle speed and torque demand at the wheels are used as reference to the set points for the control algorithms, similar to the work presented in [13] and [16]. The correlation between the SOC and the torque demand with vehicle speed is used for the EM controller. Likewise, the wheel slip and torque demand correlation is used for the traction controller with vehicle speed. The extracted desired SOC and $\lambda$ values used to determine the controller set points are shown in Figs. 12 and 13, respectively. A linear piecewise interpolation technique available in Matlab is used for all surface plots.

\section{A. Vehicle Traction Controller}

An error-based feedback controller is used for the traction control. The objective of the controller is to regulate the wheel slip. An empirical tire model is used in the complex model. An allowable wheel slip value is taken from a lookup table based on the optimal operating points. During the high torque demand cases, the vehicle is allowed to experience more wheel slip.

Fig. 14 presents the wheel slip of the vehicle when the designed traction controller is used in simulations. The dynamic weight transfer to the rear and front axles in acceleration and decelerating situations activates the traction controller. 

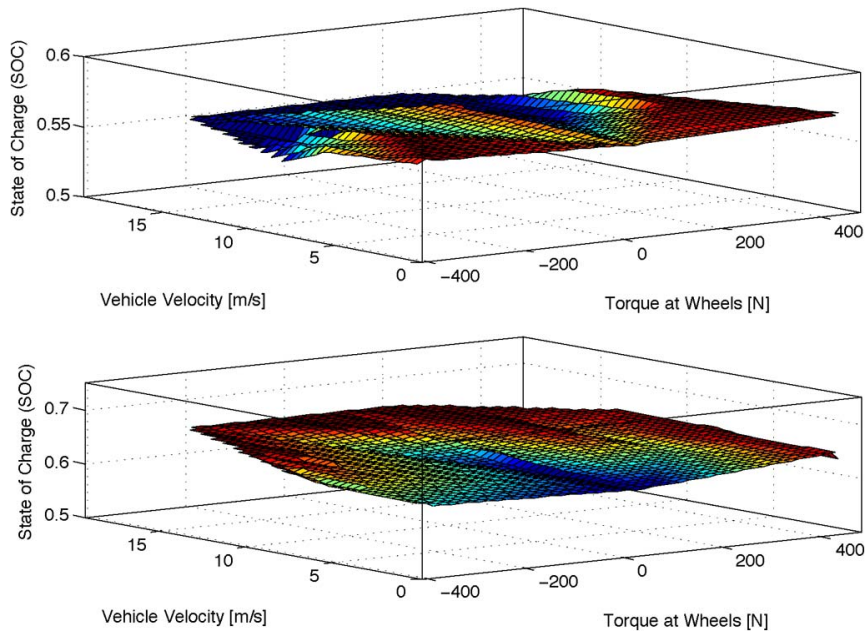

Fig. 12. Desired SOC for optimal operation surfaces [EM only (bottom) and concurrent (top)] after piecewise interpolation fit in Matlab.
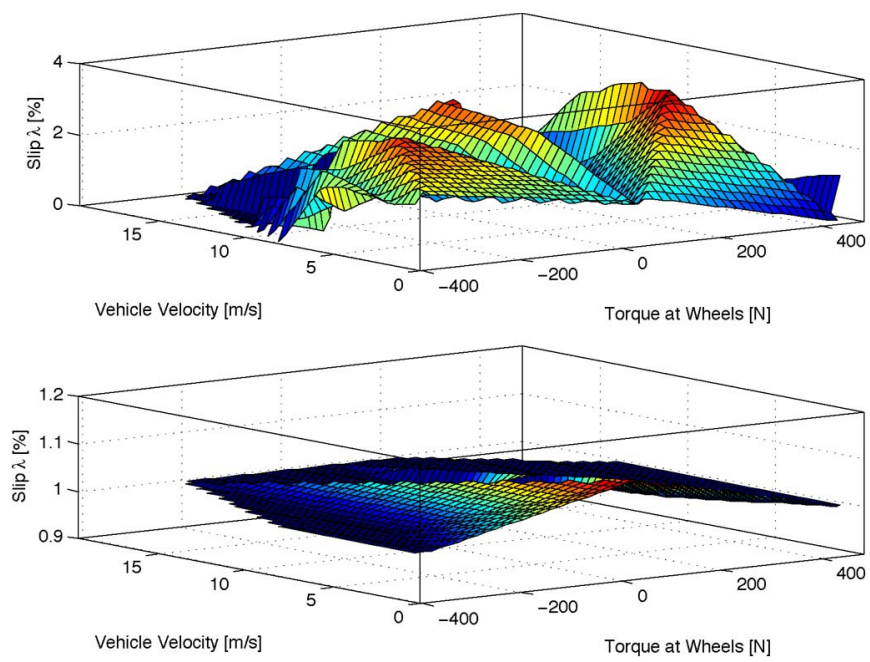

Fig. 13. Desired SOC for optimal operation points [traction controller only (bottom) and concurrent (top)] after piecewise interpolation fit in Matlab.
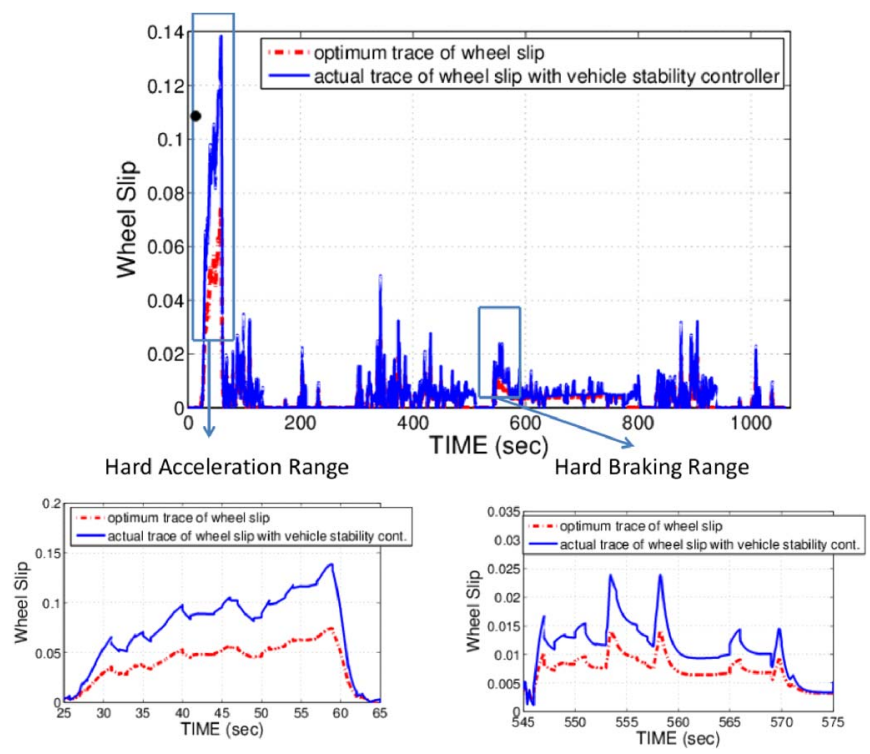

Fig. 14. Wheel slip behavior of the vehicle with a traction controller.
TABLE VI

EM CONTROLLER RULES

\begin{tabular}{|c|l|}
\hline CONDITION & OPERATING MODE \\
\hline if $\left|S O C_{\text {diff }}\right|<S O C_{\text {diff }, c r}$ & Normal Mode \\
\hline if $S O C_{\text {diff }}<-S O C_{\text {diff }, c r}$ & Charging Mode \\
\hline if $S O C_{\text {diff }}>S O C_{\text {diff,cr }}$ & Discharging Mode \\
\hline if $S O C_{\text {diff }}>S O C_{\text {diff }, c r}$ & Mechanical Braking \\
\hline
\end{tabular}

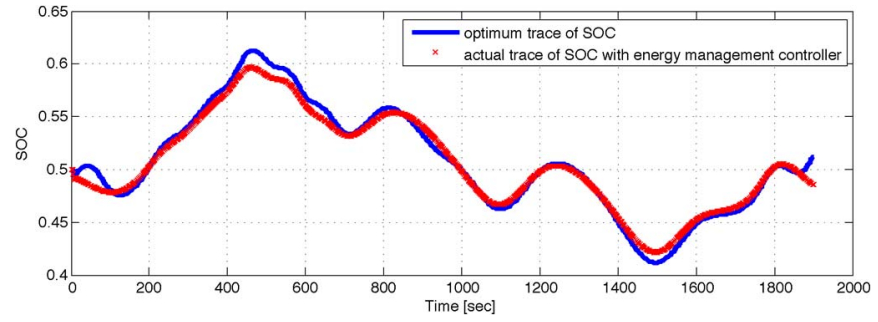

Fig. 15. SOC behavior of the vehicle with an EM algorithm versus the DP trace.

TABLE VII

CRitical Values of SOC AND Wheel SliP

\begin{tabular}{|c|c|}
\hline wheel slip $_{c r}$ & 0.03 \\
\hline$S O C_{\text {diff,cr }}$ & 0.15 \\
\hline$S O C_{\text {diff,cr } 1}$ & 0.15 \\
\hline$S O C_{\text {diff,cr } 2}$ & 0.13 \\
\hline
\end{tabular}

\section{B. EM Controller}

An EM algorithm is also designed using the DP study results. In Table VI, the rules for this algorithm are given. The controller calculates the operating mode of the powertrain based on these conditions using a lookup table. $\mathrm{SOC}_{\mathrm{diff}, \mathrm{cr}}$ represents the critical value of the difference between the actual and desired SOC values. The critical value is obtained by analyzing the controller for various cases. If the SOC difference is in the normal range, an existing controller algorithm is used. If the SOC difference is higher than a specified threshold, the system uses only mechanical brakes instead of regenerative braking for safety reasons.

The SOC trace of the vehicle battery is given in Fig. 15 using the casual EM algorithm in simulations compared with the dynamic optimization results. Same initial values are used for both cases, and the results show with the controller settings shown in Table VII that the results give similar results. It should also be noted that, in the case of overtuning to the DP results, the performance of the controller using other drive cycles deteriorates since the problem becomes response fitting than operating around the optimal conditions.

\section{Concurrent Supervisory Controller}

Using the optimal operating points presented in Figs. 12 and 13 , a concurrent controller using both control actions is developed. The rule set for the controller is summarized in Figs. 16 and 17 . The critical values of the SOC difference $\mathrm{SOC}_{\text {diff,cr1 }}$ and $\mathrm{SOC}_{\mathrm{diff}, \mathrm{cr} 2}$ change with respect to the condition of wheel slip, compared with a wheel slip value threshold wheel slip $_{\mathrm{cr}}$. Table VII shows the values used in the simulations. 


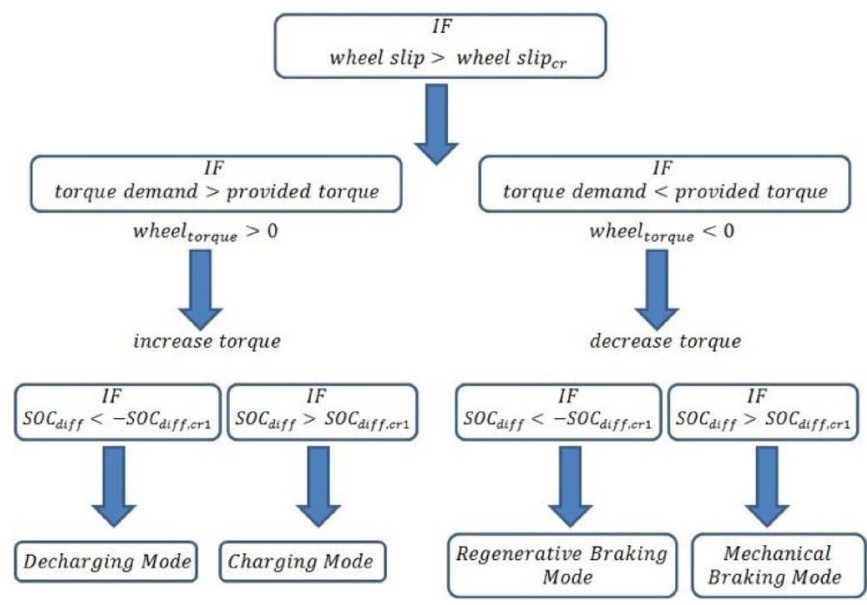

Fig. 16. Extracted rules for concurrent controller when wheel slip is high.

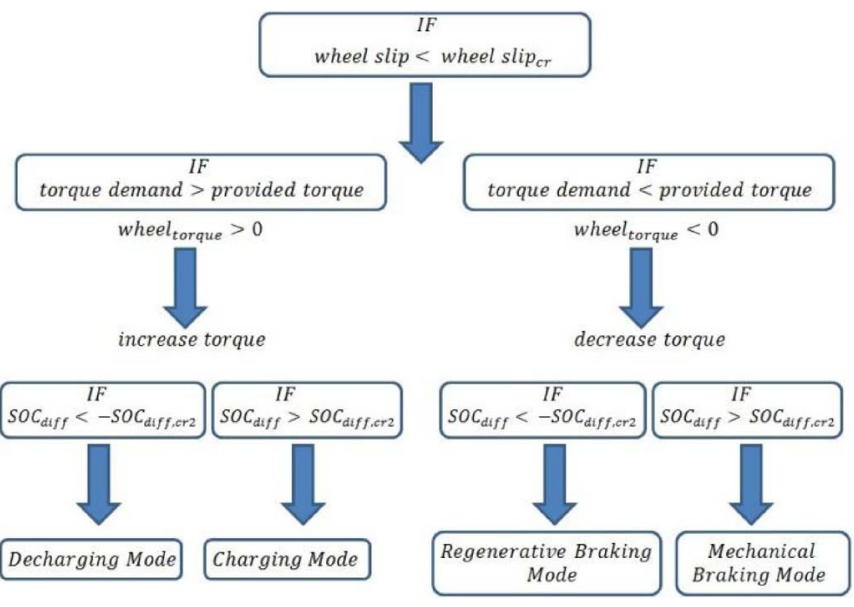

Fig. 17. Extracted rules for concurrent controller when wheel slip is low.

Figs. 18 and 19 present the SOC and the wheel slip behavior of vehicle with the developed controllers. Using a nonzero initial value for slip makes little sense; however, a different initial SOC is used from the optimal conditions as a perturbation to show the system is not overtuned as to the DP results. Both of these figures show that the concurrent controller operates the vehicle around the optimal points much closer than the individual controllers do during the hard acceleration and braking regions. This is due the increased degree of freedom in control actions using the concurrent controller.

Nearly optimal operation of the vehicle affects the fuel consumption of the vehicle. The fuel consumption rate in different cases for two drive cycles are shown in Figs. 20 and 21 for UDDS and Indian Highway drive cycles, respectively. A significant difference between individual and concurrent controllers can be observed in the hard acceleration and braking regions.

In Table VIII, the cumulative performance of the controllers for the UDDS (see Fig. 20) and the Indian Highway driving cycles (see Fig. 21) are presented. These results show that significant improvements can be obtained by using a concurrent control algorithm rather than using individual controllers, designed separately.

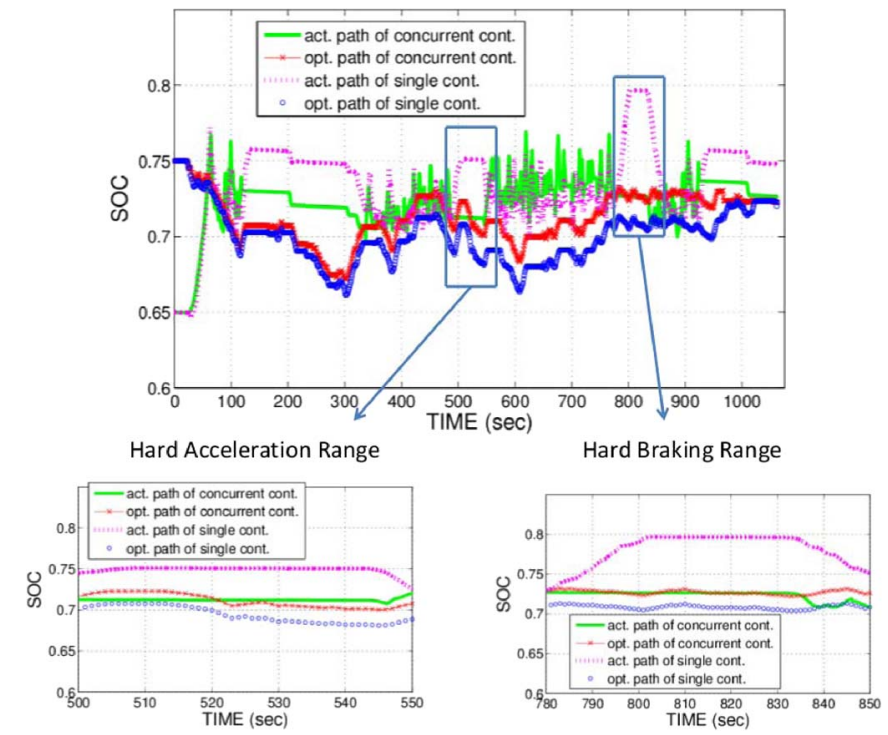

Fig. 18. SOC behavior of the concurrent controller.

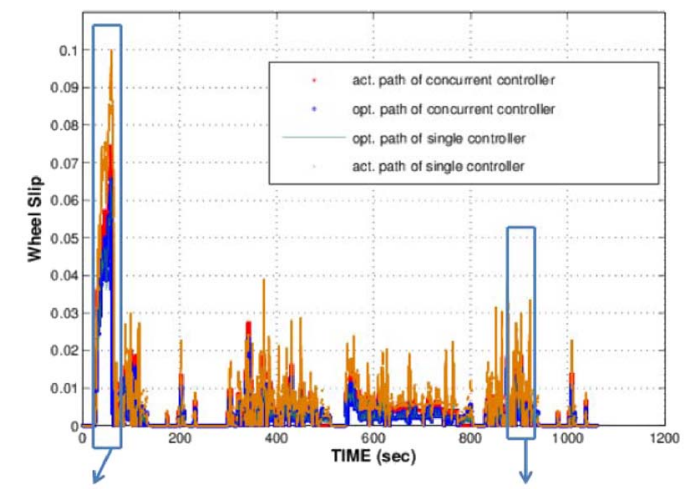

Hard Acceleration Range

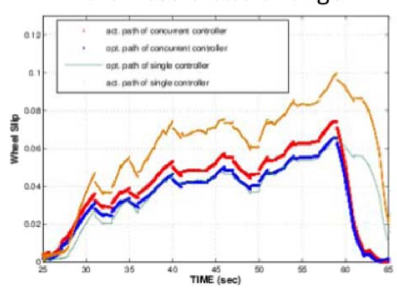

Hard Braking Range

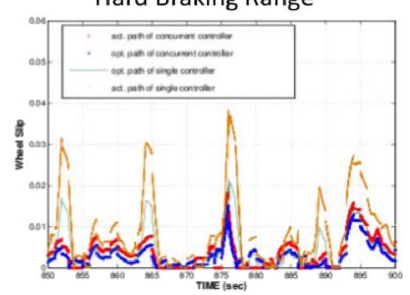

Fig. 19. Wheel slip behavior of the concurrent controller.

\section{Conclusion}

In this paper, concurrent design of EM and traction control algorithms for a vehicle equipped with a parallel hybrid powertrain and transfer case is presented. Our primary objective is to highlight the opportunities of considering the interactions between design problems in automotive control systems. Rulebased supervisory controllers are designed based on the optimal operating states obtained after solving a DP problem. The cases of using EM and traction control algorithms separately and together (i.e., concurrent control) are investigated. The performance of the concurrent controller is compared with the EM and vehicle stability controllers that are designed separately. Under the same conditions, the vehicle with the concurrent supervisory controller is $16.47 \%$ more efficient in fuel 


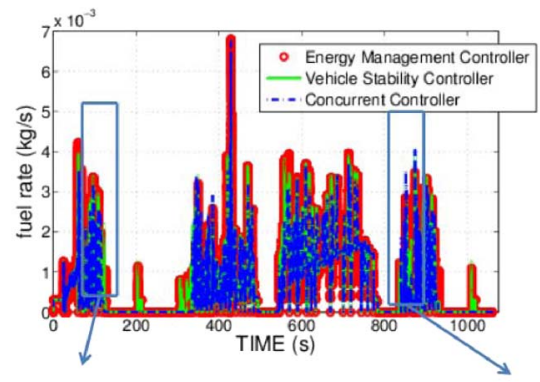

Hard Acceleration Range

Hard Braking Range
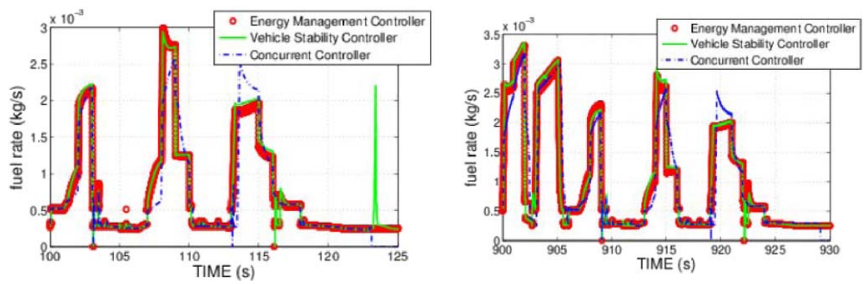

Fig. 20. Fuel rate behavior of the concurrent controller with UDDS.

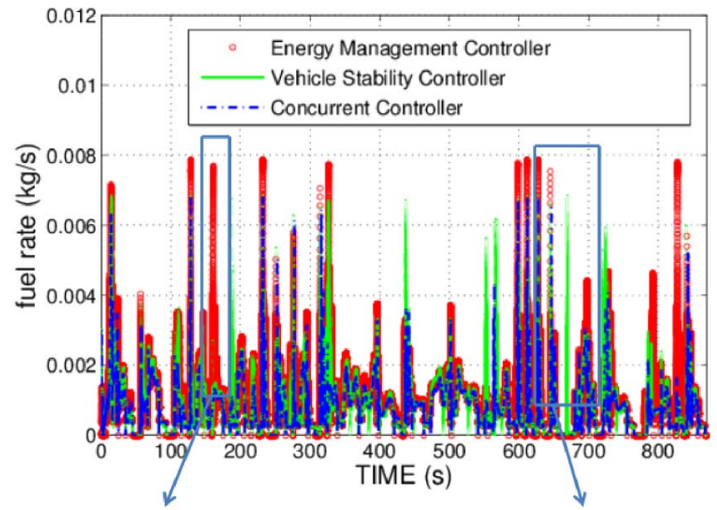

Hard Acceleration Range

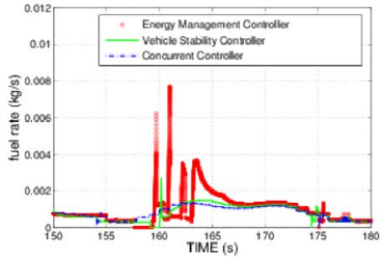

Hard Braking Range

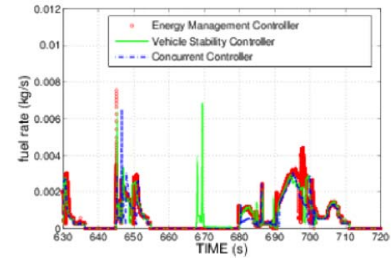

Fig. 21. Fuel rate behavior of the concurrent controller with Indian highway.

TABLE VIII

FUEL CONSUMPTION AND WHEEL SLIP COMPARISON

\begin{tabular}{|c|c|c|c|c|}
\hline & Fuel & Average & \multicolumn{2}{|c|}{ Improvement } \\
& $\begin{array}{c}\text { Cons. } \\
(l / 100 k m)\end{array}$ & $\begin{array}{c}\text { Slip } \\
(\%)\end{array}$ & $\begin{array}{c}\text { Fuel } \\
\text { Cons. }\end{array}$ & $\begin{array}{c}\text { Average } \\
\text { Slip }\end{array}$ \\
\hline EM & 8.5 & & & \\
\hline VSC & & 3.7 & & \\
\hline $\begin{array}{c}\text { Concurrent } \\
\text { Controller }\end{array}$ & 7.1 & 3.25 & $16.47 \%$ & $12.16 \%$ \\
\hline
\end{tabular}

consumption and experiences $12.16 \%$ less wheel slip, assuming slippery road friction conditions.

The improvements shown in this paper is valid under described conditions assumed for the simulation model. The method presented here uses a commonly accepted drive cycle to identify the optimal operation points of the vehicle. The profile of the drive cycle used in the DP is also important to identify the number of optimal operation points identified and should be considered during the design phase. Our rulebased supervisory controller sets desired SOC and slip values, which are the optimal values for the vehicle based on torque demanded at the current speed. The better (more realistic) these optimal points are identified, the better our resulting controller will be. The variation of mass and center of mass location can also affect the performance of the controllers. By using pressure sensor information of each tire, overall mass and loading conditions can be predicted to update the controller calibration. Our future work includes using more than two control problems, solving the optimization problem using a stochastic formulation, and studying the sensitivity of the design method to the drive cycle content during the optimization phase.

\section{REFERENCES}

[1] D. Kim, S. Hwang, and H. Kim, "Vehicle stability enhancement of fourwheel-drive hybrid electric vehicle using rear motor control," IEEE Trans. Veh. Technol., vol. 57, no. 2, pp. 727-735, Mar. 2008.

[2] F. Borelli, A. Bemporad, M. Fodor, and D. Hrovat, "An MPC/hybrid system approach to traction control, IEEE Trans. Control Syst. Technol., vol. 14, no. 3, pp. 541-552, May 2006.

[3] Y. Huang, C. Yin, and J. Zhang, "Optimal torque distribution control strategy for parallel hybrid electric buses," WSEAS Trans. Syst., vol. 7, no. 6, pp. 758-773, Jun. 2008.

[4] C. Lin, H. Peng, and J. W. Grizzle, "A stochastic control strategy for hybrid electric vehicles," in Proc. Amer. Control Conf., 2004, vol. 5, pp. 4710-4715.

[5] K. Tong, "Simultaneous plant/controller optimization of traction control for electric vehicle," M.S. thesis, Dept. Elect. Comput. Eng., Univ. Waterloo, Waterloo, ON, Canada, 2007.

[6] S. Delprat, J. Lauber, T. M. Guerra, and J. Rimaux, "Control of a parallel hybrid power train: Optimal control," IEEE Trans. Veh. Technol., vol. 53, no. 3, pp. 872-881, May 2004.

[7] S. G. Wirasingha, S. M. Lukic, F. Rodriguez, A. I. Antoniou, and A. Emadi, "Adaptive control for hybrid electric vehicles," Int. J. Elect. Hybrid Veh., vol. 3, no. 2, pp. 872-881, 2011.

[8] A. Sciaretta and L. Guzzella, "Control of hybrid electric vehicles," IEEE Control Syst. Mag., vol. 27, no. 2, pp. 60- 70, Apr. 2007.

[9] A. Rousseau, "Psat training Part 05," Argonne Nat. Lab., Lemont, IL, USA, Sep. 2011. [Online]. Available: http://www.transportation.anl.gov/ pdfs/HV/406.pdf

[10] N. J. Schouten, M. A. Salman, and N. A. Kheir, "Fuzzy logic controller parallel hybrid vehicles," IEEE Trans. Veh. Control Syst. Technol., vol. 10, no. 3, pp. 460-468, May 2002.

[11] A. G. Ulsoy, H. Peng, and M. Çakmakci, Automotive Control Systems. Cambridge, U.K.: Cambridge Univ. Press, 2012.

[12] L. Serrao, S. Onori, and G. Rizzoni, "A comparative analysis of energy management strategies for hybrid electric vehicles," J. Dyn. Syst., Meas., Control, vol. 133, no. 3, May 2011, Art. ID. 031012.

[13] D. Pei and M. J. Leamy, "Dynamic programming-informed equivalent cost minimization control strategies for hybrid-electric vehicles," J. Dyn. Syst., Meas., Control, vol. 135, no. 5, Jun. 2013, Art. ID. 051013.

[14] G. Panzani et al., "Designing on-demand four-wheel-drive vehicles via active control of the central transfer case," IEEE Trans. Intell. Transp. Syst., vol. 11, no. 4, pp. 931-941, Dec. 2010.

[15] M. Amodeo, A. Ferrara, R. Terzaghi, and C. Vecchio, "Wheel slip control via second-order sliding-mode generation," IEEE Trans. Intell. Transp. Syst., vol. 11, no. 1, pp. 122-131, Mar. 2010.

[16] H. Z. Li et al., "PID plus fuzzy logic method for torque control in traction control system," Int. J. Autom. Technol., vol. 13, no. 3, pp. 441-450, Mar. 2012.

[17] W. Kirchner and S. Southward, "Implementation and verification of adaptive longitudinal traction control," Veh. Syst. Dyn., vol. 51, no. 11, pp. 1674-1694, Nov. 2013. 
[18] J. Kang, J. Yoo, and K. Yi, "Driving control algorithm for maneuverability, lateral stability, and rollover prevention of $4 \mathrm{WD}$ electric vehicles with independently driven front and rear wheels," IEEE Trans. Veh. Technol., vol. 60, no. 7, pp. 2987-3001, Sep. 2011.

[19] M. Cakmakci and A. G. Ulsoy, "Swappable distributed mimo controller for a VCT engine," IEEE Trans. Control Syst. Technol., vol. 19, no. 5, pp. 1168-1177, Sep. 2011.

[20] H. I. Dokuyucu and M. Cakmakci, "Concurrent design of energy management and vehicle stability algorithms for a parallel hybrid vehicle using dynamic programming," in Proc. ACC, 2012, pp. 535-540.

[21] Autonomie Website.

[22] D. P. Bertsekas, Dynamic Programming and Optimal Control. vol. 1, 3rd ed. Belmont, MA, USA: Athena Scientific, 2005.

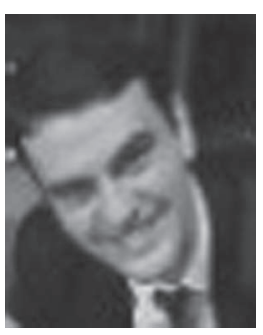

Halil I. Dokuyucu is currently working toward the graduate degree with the Department of Mechanical Engineering, Bilkent University, Ankara, Turkey.

His research interests include dynamic systems and control theory and their applications to vehicular transportation systems.

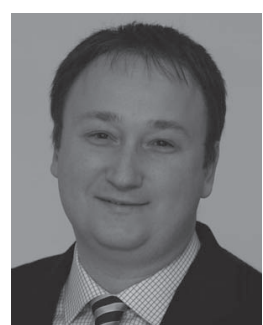

Melih Cakmakci (M'14) received the B.S. degree in mechanical engineering from Middle East Technical University, Ankara, Turkey, in 1997 and the M.S. and $\mathrm{Ph} . \mathrm{D}$. degrees in mechanical engineering from the University of Michigan, Ann Arbor, MI, USA, in 1999 and 2009, respectively.

$\mathrm{He}$ is currently an Assistant Professor of mechanical engineering with Bilkent University, Ankara, Turkey. Prior to joining Bilkent University, he was a Senior Engineer with the Ford Scientific Research Center. His research interests include modeling, analysis, and control of dynamic systems, control systems, smart mechatronics, and modeling of manufacturing systems and their control, automotive control systems, optimal energy-management algorithms, and design and analysis of network control systems.

Dr. Cakmakci is a member of the American Society of Mechanical Engineers and the Society of Automotive Engineers. 